\title{
A new method for evaluating the influences of surface topography on fatigue propriety of the random machined surfaces
}

\author{
Lin $\mathrm{Ma}^{1,2}$, GuangTao Xu ${ }^{1,2 *}$, Gang Wang ${ }^{1,2}$, and MingHao Zhao ${ }^{1,2,3}$ \\ ${ }^{1}$ School of Mechanical Engineering, Zhengzhou University, Zhengzhou, 450001, China \\ ${ }^{2}$ Henan Key Engineering Laboratory for Anti-fatigue Manufacturing Technology, Zhengzhou, 450001, China \\ ${ }^{3}$ School of Mechanics \& Engineering Science, Zhengzhou University, Zhengzhou, Henan, 450001, China
}

\begin{abstract}
The Stress concentration factor (SCF) induced by the machined surface is more complex than that resulting from macro-geometry discontinuities and has great effect on fatigue life of structure. However, another important parameter, stress gradient (SG), was always ignored. The notch roots or valleys of the wave surface constitute fatigue hot points, where cracks occur, so it is essential to study the SCF and SG at valleys rather than just the root-mean-square SCF variable. In this work, a new method for evaluating the influences of surface topography on fatigue propriety of the random machined surfaces was given. An analytical method using Fourier transformation to simulate machined surface topography is presented. Analytical formulae for SCF and SG for random machined surfaces are derived subjected to a general loading and validate these formulae via finite element method (FEM). Joint probability-distribution function for SCF and SG at the valleys of the random machined-surface topography of the machined sample was obtained after different cycles fatigue test. This method gave us how the surface topography effect the fatigue properties of machined components. Fatigue test of machined sample for a single crystal nickel based alloy were established for validated this method. The obtained results should be useful in studying and evaluating fatigue properties of machined components.
\end{abstract}

\section{Introduction}

The stress concentration factor ( $\mathrm{SCF}$ ) is an important parameter in the application of mechanical engineering and knowledge of this parameter and the stress distribution around notches is important for engineers engaged in the design or fatigue-life prediction of engineering components [1-4].

However, the stress concentration induced by the machined surface is more complex than that resulting from macro-geometry discontinuitiesand is characterized by the occurrence of (among other features) multiple hotspots. Neuber's rule characterized stress concentrations introduced by machined surface topography [3]. Castagnetti and Dragoni [5] proposed a method for modifying this rule, by changing the expression of the depth-reduction factor, to increase the range of applicability and improve the corresponding accuracy. Gao [6] analyzed the stress concentration for slightly undulating surfaces by employing elastic Green's functions. The first-order solution of the SCF was obtained by treating the surface as a perturbation from a reference plane. Recently, Medina [7] derived a stress concentration formula for an arbitrary shallow surface using the first-order perturbation approach, which can be applied in various cases (including undulating surfaces, parabolic notches, and Gaussian notches) according to Rice [8], for the analysis of the stress intensity resulting from a varying crack front. Medina and Hinderliter [9] obtained an analytical solution for the SCF of a slightly roughened random surface by combining Gao's method, the Hilbert transform and the energy conservation principle.

Stress concentrations induced by the surface topography have a significant effect on the fatigue life of structures $[10,11]$. The Stress Gradient (SG) near the stress concentration may also influence the fatigue life of components [4, 12-14]. The relative SG ahead of notches, as calculated (with a high degree of accuracy) by Filippini [15], exhibited a strong dependence on only the notch radius. The volumetric method can be used to determine the fatigue life from the relative SG [16]. Moreover, the influence of the size effect and the SG on the high-cycle fatigue strength of a DIN EN 10088-3 martensitic stainless steel has been experimentally determined and simulated via the finite element method [17]. The corresponding results confirmed that SG has a significant effect on the fatigue strength.

There are many roughness parameter used to evaluate the fatigue life[18], such as, the average roughness ( $\mathrm{Ra})$, peak to valley height roughness (Ry) and10-points roughness $(\mathrm{Rz})$ to characterize roughness of a machined surface. And surface quality is positive with increasing fatigue cycles. However, the reduction in fatigue life with increasing $\mathrm{Ra}$ did not occur in a consistent manner $[19,20]$. The fatigue stress concentration factor, related to $\mathrm{SG}$ and $\mathrm{SCF}$, can describe the relationship between surface topography and fatigue life. Hovever, there was few work for using the $\mathrm{SC}$ and $\mathrm{SG}$ to evaluate the

Corresponding author: xgtzzu@zzu.edu.cn, xgtzzu@163.com 
influences of surface topography on fatigue propriety of the random machined surfaces.

Therefore, SG and SCF, which both are dependent on the surface topography of the machined surface, must be simultaneously considered during fatigue-property evaluation. As such, in the present work, we propose a method to evaluate the influences of surface topography on fatigue propriety of the random machined surfaces. This paper is organized as follows: an approach for simulations of the machined surface from the measured data was given in the section 2 , the analytical solutions of SCF and SG, obtained via Fourier transformation in section 3 , in the section 4 , validation of these solutions under uniform loading via finite element simulations was presented, a 2D joint probability-distribution function of SCF and SG associated with the machined-surface topography was given in section 5 , different fatigue test cycles for the proposed method to evaluate the influences of surface topography on fatigue propriety of the random machined surfaces in the section 6. Finally, the conclusions of the study are presented.

\section{Analytical simulation of random machined surfaces}

The random machined-surface topography can be determined by measuring a series of discrete data points corresponding to location and height, using a BRUKER NPFLEX 3D Optical Microscope (vertical resolution: 3 $\mathrm{nm}$.) The topography is expressed by superposing a series of cosine components on the surface as follows [21]:

$$
y(x)=\sum_{i=1}^{n} a_{i} \cos \left(\frac{2 \pi x}{\lambda_{i}}+\theta_{i}\right)
$$

where $y(x)$ is the measured surface topography, $\mathrm{n}$ is the number of series components, and $a_{i}, \lambda_{i}$ and $\theta_{i}$ are the amplitude, wave-length, and phase angle, respectively, of the cosine series. Using a FFT algorithm code in MATLAB, we can transform the measured topography into a cosine series with known parameters $a_{i}, \lambda_{i}$ and $\theta_{i}$

Determining the number of cosine components as shown in Eq.(1) at each point is essential for accurate characterization of the topography. We denote the measured machined-surface topography and the simulated-surface topography as $R(x)$ and $W(x)$, respectively. The difference between these topographies decreases with increasing number of terms in the series, and extremely high frequency components may be neglected, owing to the presence of the noise signal. We defined the following criterion:

$$
\left|\frac{R(x)-W(x)}{R(x)}\right|<\delta
$$

where $\delta$ is the preset relative error between the original measured machined surface and the simulated surface topography. In this paper, we assume that $\delta=1 \%$.

As Fig. 1 shows, the simulated surface topography corresponds closely to the measured original machinedsurface topography.

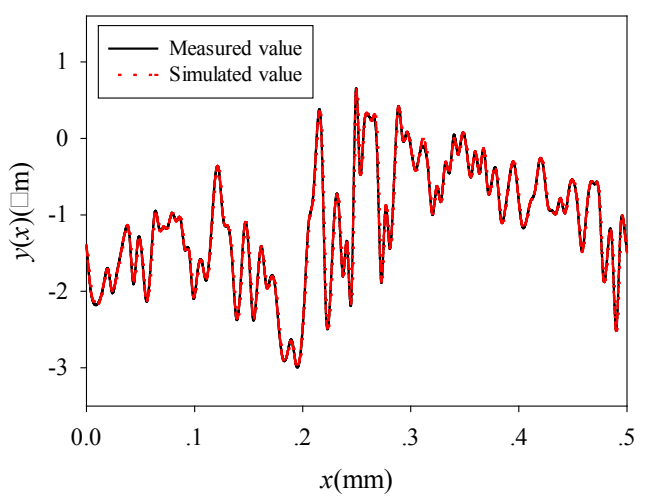

Fig.1. Measured surface topography and simulated surface topography.

\section{Stress concentration factor and stress gradient of surface topography}

Gao [6] conducted a first-order perturbation analysis of a slightly undulating surface of a half plane subjected to uniform tension $T$ and obtained the following expression for the stress:

$$
\begin{aligned}
& \sigma_{x x}(x, y)=T\left\{1+\frac{2}{\pi}\right. \\
&\left.\int_{-\infty}^{\infty} \operatorname{Re}\left[\frac{x^{\prime}-x}{\left(x^{\prime}-x-i[y-a(x)]\right)^{3}}\right]\left[a\left(x^{\prime}\right)-a(x)\right] d x^{\prime}\right\},
\end{aligned}
$$

where $a(x)$ : random surface and $|y-a(x)|$ : vertical distance from the observation point $(x, y)$ to the surface $y=a(x)$.

Using Fourier transformation, the random surface can be described by Eq. (1) and substituting this expression into Eq. (3), yields get the stress component $\sigma_{x x}(x, d)$ acting at a depth $d$ from the surface:

$$
\sigma_{x x}(x, d)=T\left[1+\sum_{i=1}^{n} \frac{4 \pi a_{i}}{\lambda_{i}}\left(1-\frac{\pi d}{\lambda_{i}}\right) e^{-\frac{2 d \pi}{\lambda}} \cos \left(\frac{2 \pi x}{\lambda_{i}}+\theta_{i}\right)\right]
$$

By inserting $d=0$ into Eq. (4), we obtain the analytical expressions for SCF and SG at any point on the random surface. These expressions are given as follows: 


$$
\begin{aligned}
& K_{t}(x)=\frac{\sigma_{x x}}{T}=1-4 \pi \sum_{i=1}^{n} \frac{a_{i}}{\lambda_{i}} \cos \left(\frac{2 \pi x}{\lambda_{i}}+\theta_{i}\right), \\
& \chi(x)=\frac{\partial \sigma_{x x}}{\partial d} / T=-12 \pi^{2} \sum_{i=1}^{n} \frac{a_{i}}{\lambda_{i}^{2}} \cos \left(\frac{2 \pi x}{\lambda_{i}}+\theta_{i}\right) .
\end{aligned}
$$

The SCF obtained for a single cosine wave with $a / \lambda<0.1$, differed a maximum of $8 \%$ from that determined via the finite element method [22]. Eqs. (5a) and $(5 b)$ are based on and, hence, has the same precision as Eq. (3).

\section{Validation of the analytical solutions via finite element simulations}

\subsection{SCF and SG under non-uniform loading}

As a numerical example for the section 3, we consider a half plane with a machined surface, subjected to a linear load, $F(y)$, as shown in Fig. 2, to validate the analytical solutions (5a) and (5b). Non-uniform loading (such as a bending load) is frequently encountered in engineering activities. Therefore, the effect of linear loading on the SCF and SG of machined surface must be determined.

The surface amplitude is negligible compared with the characteristic size of the components. Therefore, the SCF is assumed to be the same as that associated with uniform stress, and is described by the first-order solution given in Eq. (5a), where

$$
T=F(0),
$$

whereas the SG is calculated from:

$$
\chi_{B}=\chi_{T}+\chi_{\sigma}
$$

where $\chi_{T}$ is the SG associated with a uniform stress load, and $\chi_{\sigma}$ is the gradient of the applied linear load

$$
\chi_{\sigma}=\frac{1}{T}\left(\left.\frac{d F(y)}{d y}\right|_{y=0}\right)
$$

For the single wave, the SCF (Fig. 3) and SG (Fig. 4) are determined via the analytical formulae and the finite element method. Furthermore, maximum relative error values of $8 \%$ and $3 \%$ for the SCF and SG, respectively, are realized when the gradient of the applied linear load is $2 \mathrm{~mm}^{-1}$.

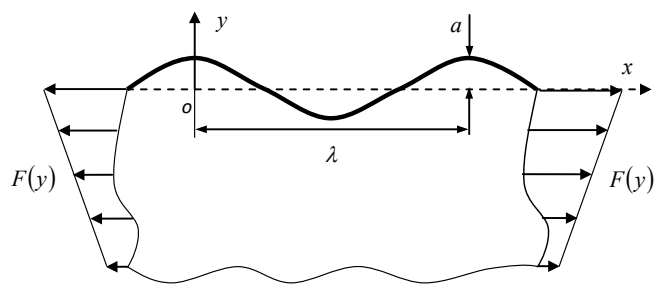

Fig.2. Single cosine wave surface schematic model under nonuniform loading.

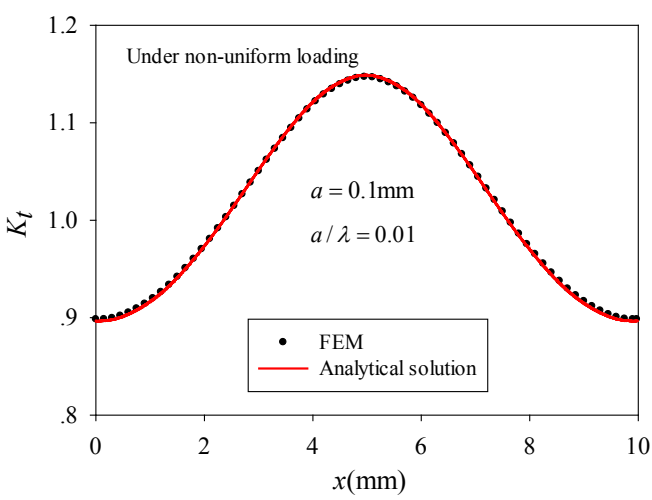

Fig.3. Stress concentration factor under non-uniform loading.

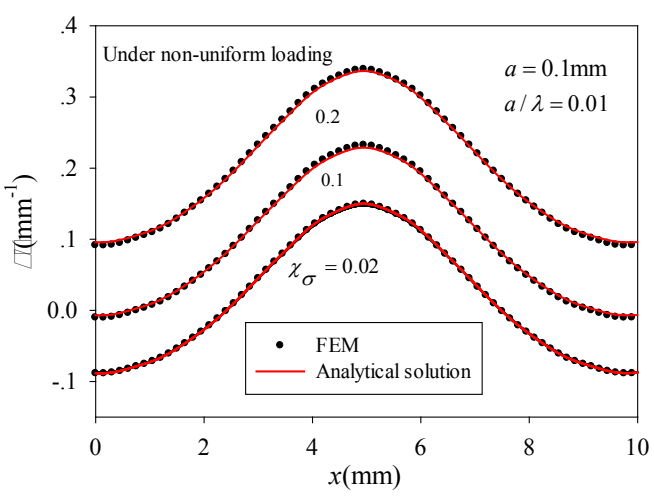

Fig.4. Stress gradient under non-uniform loading.

\subsection{Random machined surfaces}

The stress of a semi-infinite plate with the measured machined surface was determined following the procedure described in section 3. The SCF (Fig. 5) and SG (Fig. 6) were determined by the analytical solution and via finite element model with a triangular-element mesh. The close correspondence obtained verifies that the analytical formula, see Eq. (5), can be used to calculate the SCF and SG induced by random machinedsurface topography. 


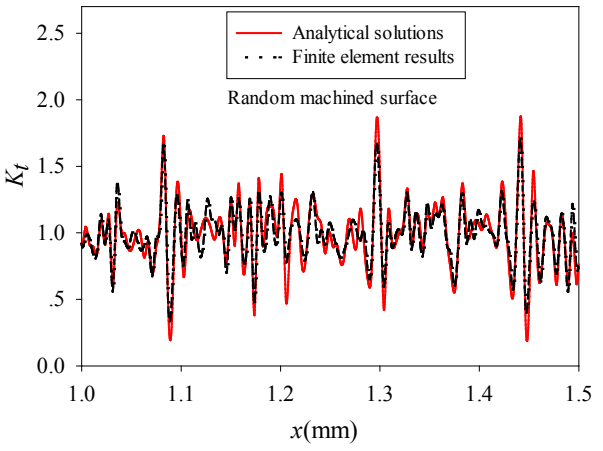

Fig.5. Stress concentration factor of the machined original surface.

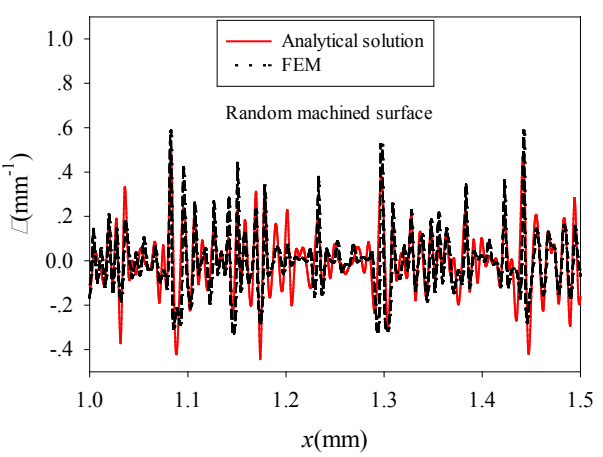

Fig.6. Stress gradient of the machined original surface.

\section{Two-dimensional joint probability distribution function for SCF and SG of machined-surface topography}

As reported in a previous study, the notch root or valley of the wave surface constitutes a fatigue hot point, where cracks occur [23, 24]. Therefore, we simultaneously consider the stress concentration at the valleys along the random surface, and the SG rather than just the singlerandom variable SCF.

We measured eight groups of the 2D machined surface ( $4 \mathrm{~mm}$ length with $R a=0.2 \mu \mathrm{m}$ ), which are perpendicular to the direction of the machining grooves. To prevent the effects associated with inhomogeneity, we chose an evaluation length of $4 \mathrm{~mm}$, in accordance with the ISO 4288-1996 standard. The SCF and SG of the machined surface topography were obtained using the respective analytical formulae (see Eq. (5)).

After assuming a normal distribution, for both random variables, we then determined the relativity by calculating the covariance matrix. The covariance of both random variables is always $>0$. Using mathematical statistics [25], we determined the 2D normal density function by calculating the mean, variance, standard deviation, and relative parameter of the samples. The 2D normal density function is given as follows:

$$
\begin{gathered}
f\left(K_{t}, \chi\right)=\frac{1}{2 \pi \sigma_{1} \sigma_{2} \sqrt{1-\rho^{2}}} \exp \left\{-\frac{1}{2\left(1-\rho^{2}\right)}\right. \\
\left.\left[\left(\frac{K_{t}-\mu_{1}}{\sigma_{1}}\right)^{2}-2 \rho \frac{K_{t}-\mu_{1}}{\sigma_{1}} \cdot \frac{\chi-\mu_{2}}{\sigma_{2}}+\left(\frac{\chi-\mu_{2}}{\sigma_{2}}\right)^{2}\right]\right\},
\end{gathered}
$$

in this example, the variance $\sigma_{1}{ }^{2}=0.0786, \sigma_{2}{ }^{2}=0.0511$ , mean $\mu_{1}=1.34, \mu_{2}=0.281$, and relative coefficient $\rho=0.906$ (see Fig. 7).

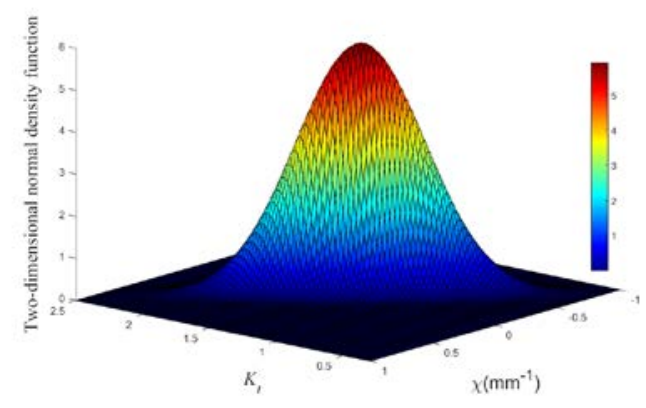

Fig. 2 Two-dimensional normal density function.

\section{Evolution the influences of surface topography on fatigue propriety of the random machined surfaces}

In order to further investigate how the surface topography effect on fatigue life and simultaneously introduce SCF and SG these two-dimensional random variable, we did SC nickel-based super alloy fatigue test in the same environment with different surface topography, decreasing grain boundary and material microstructure influence factors as far as possible.

\subsection{Specimen preparation}

Cylindrical test specimens, as shown in Fig. 8, have been machined by grinding with lubricant. Test specimens were prepared in such a way that maximum load is applied perpendicular to the rolling direction. To characterize roughness of a machined surface, average roughness $(\mathrm{Ra})$ is generally used. The machined surfaces $(R a=0.4 \mu \mathrm{m})$ specimen, were produced by varying the feed rate. Measurement surface topography of every specimen sign region, which is $4 \mathrm{~mm}$ in length in the middle part of the sample, as shown in Fig. 8 red box, still used a BRUKER NPFLEX 3D Optical Microscope as initial surface topography.

\subsection{Experimental procedures}

The tensile strength properties of the SC, as shown in Table 1, were obtained from specimens prepared according to ASTM E-8 Standard [26]. The tensile tests 
were performed at a strain rate of $0.001 / \mathrm{s}$ in a $15 \mathrm{kN}$ servo-hydraulic MTS machine.

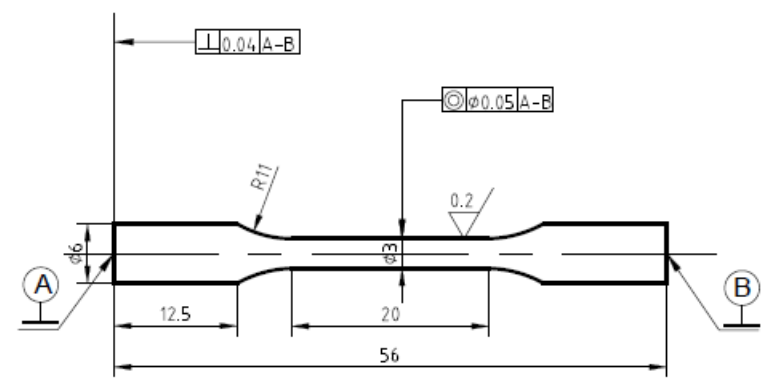

Fig.3.Specimen geometry (dimensions in $\mathrm{mm}$ ).

Table 1 Tensile mechanical properties of SC nickel-based superalloy.

$\frac{\frac{\sigma_{Y S}(\mathrm{MPa})}{\sigma_{U T S}(\mathrm{MPa})}}{963}$

Axial fatigue tests have been performed at frequency of $6 \mathrm{~Hz}$ in ambient conditions utilising a loading ratio of $R=0.1$ under sinusoidal waveform at room temperature according to ASTM E-466 [27]. The specimen were under load controlled condition using above MTS machine, respectively $0.5 \sigma_{Y S}$ and $0.4 \sigma_{Y S}$, which was expected to result in a fatigue life of between $10^{4}$ and $10^{6}$ cycles. During the cyclic testing, the specimen with $2 \mathrm{e}+5$ cycles, $3 \mathrm{e}+5$ cycles, $4 \mathrm{e}+5$ cycles, $5 \mathrm{e}+5$ cycles. When fracture occured in the effective location and tests were stopped if the specimen did not fail at $10^{7}$ cycles, the number of cycles to failure was recorded.

\subsection{Evolution of the surface topography}

By observing the same region of the specimen, Fig.9 shows surface topography was changing with cycles increasing, where $x$ is positions and $z$ is heights of the surface. Fig. 10 and Fig.11 show the evolutions of SCF and $\mathrm{SG}$, and linear regression curves increase with the cycles as a function of the number of fatigue cycles. SCF and SG was positive with increasing fatigue cycles, which might causally be involved peaks and valleys as the same reason in [28].

Form Fig.12, Fig.13 and Fig.14 we observed parameters the variance, the mean, and the relative coefficient in Eq.(8) evolution. The average of SCF and SG were increasing with fatigue cycles, however, SCF increased more quickly than $\mathrm{SG}$. The relative coefficient always kept the value of 0.9 , which showed SCF and SG has sustained high relativity.

\section{Conclusions}

An efficient approach, was developed for simulating the surface topography of random machined rough surfaces.
The SCF and SG induced by the original machined surface are essential for characterizing the surface

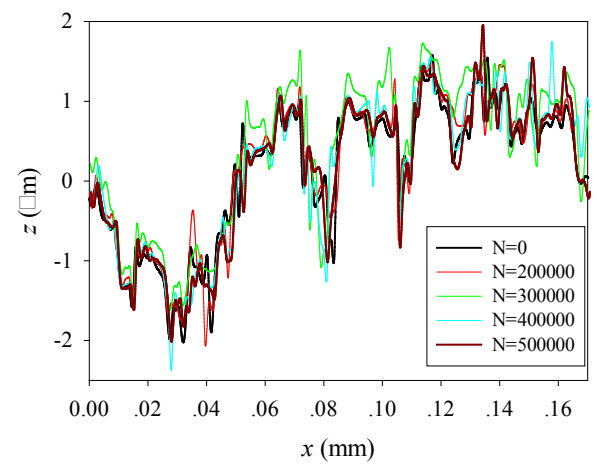

Fig.4. Surface topography for the different fatigue test cycles of the specimen.

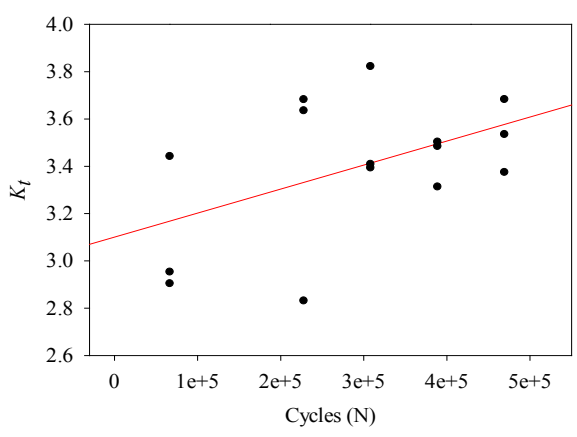

Fig.5. The SCF for the different fatigue test cycles.

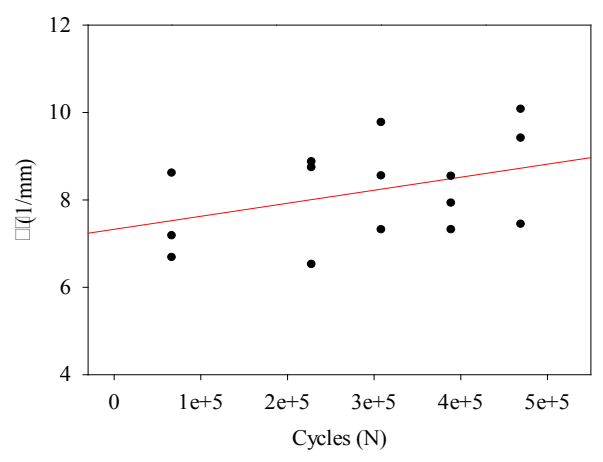

Fig.6. The SG for the different fatigue test cycles.

topography and, hence, analytical formulae for these parameters were presented. The accuracy of these formulae was validated via finite element analyses for the case of non-uniform loading, such as when linear loads are applied. We also determined the twodimensional joint probability-distribution functions for SCF and SG at the valleys along the random surface of the machined-surface topography. Finally, we eliminate the variance, the mean, and the relative coefficient as far as possible, in fatigue testing to study the evolution of 
surface topography on fatigue life. The obtained results should be useful in studying and evaluating fatigue properties of machined components.

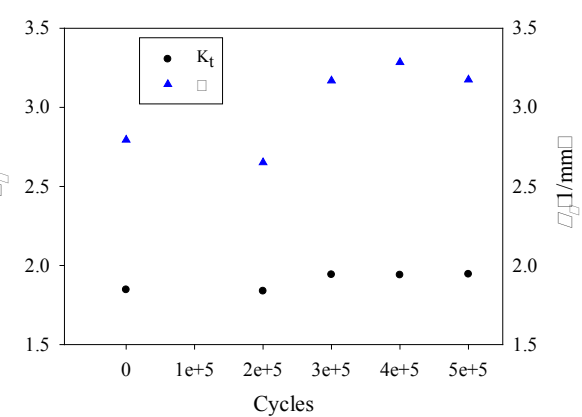

Fig. 7 The mean for the different fatigue test cycles.

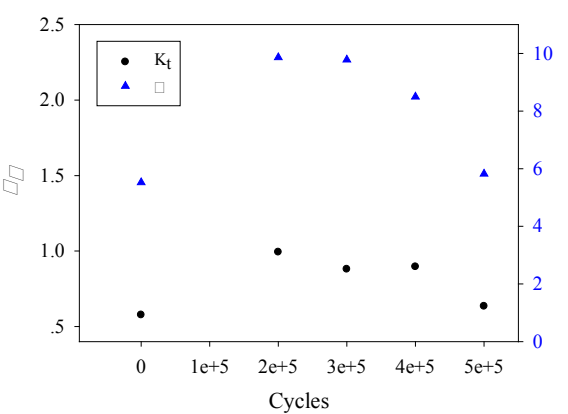

Fig. 8 The variance for the different fatigue test cycles .

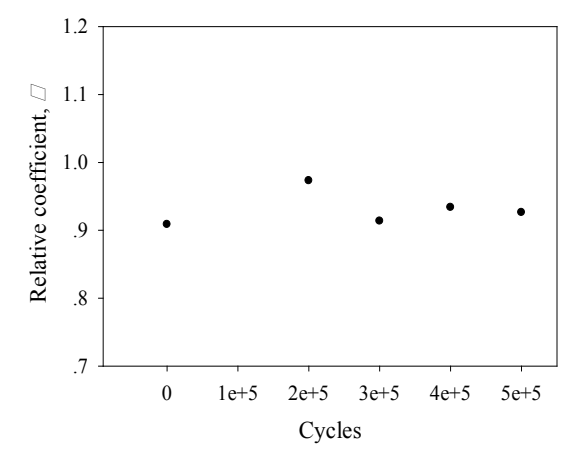

Fig. 9 Relative coefficient for the different fatigue test cycles

\section{Acknowledgements}

This work was supported by the Key Research Project of Higher Education Institution of Henan Province (No 16A460024).

\section{References}

1. Medina HE, Pidaparti R, Hinderliter B. Appl. Mechs. Rev. 67: 010802( 2014)

2. Pilkey W, Pilkey D. Peterson's Stress Concentration Factors. 3rd ed., New York :Wiley.( 2008)

3. Neuber H. Kerbspannungslehre.2nd ed., SpringerVerlag, Berlin: Germany.( 1958)
4. Peterson RE, Stress concentration factors. New York :Wiley( 1974)

5. Castagnetti D, Dragoni E. Materialwissenschaft Und Werkstofftechnik.44: 364-71.( 2013)

6. Gao H. J Mech. Phys. Solids.39: 443-58 ( 1991)

7. Medina H. Int. J. Solids. Struct. 69-70:86-93(2015)

8. Rice JR. J Appl. Mech. 52: 571, (1985)

9. Medina H, B Hinderliter. Int. J. Solids. Struct. 51 (10) :2012-2018,(2014)

10. Taylor D, Clancy OM. Fatigue. Fract. Eng. M.14: 329-36.( 2007)

11. Ås SK, Skallerud B, Tveiten BW. Int. J. Fatigue . 30: 2200-2209(2008)

12. Schijve J. Fatigue. Engng. Mater. Struct. 3: 325338( 1980)

13. Gates N, Fatemi A. Theor. Appl. Fract. Mec.84:325(2016)

14. Schijve J. Fatigue Engng Mater Struct 1982;5: 7790.

15. Filippini M. Int. J. Fatigue. 22:397-409.( 2000)

16. Adib-Ramezani H, Jeong J. Comp. Mater. Sci. 39: 649-63(2007)

17. Milošević I, Winter G, Grün F. IProcedia Eng. 160:61-68(2016)

18. ZHANG G, LIU Jun, Liu YS, Yue ZF.Journal of mechanical strength,32(1):110-115.(2010)

19. Yao CF, WU DX, Jin QC , et al. Trans. Nonferrous Met. Soc. China.23:650-660( 2013)

20. Ardi DT, Li YG, Chan KHK, Blunt L, Bache MR. Procedia Cirp.13:19-24(2014)

21. Aoto Y, Noguchi H. Int. J. Fract. 131:59-78(2005)

22. Gao H. Int. J. Solids. Struct. 28:703-25(1991)

23. Rusk DT, W Hoppe, W Braisted, N Powar. Int. J. Fatigue. 31:1454-63( 2009)

24. Smith RA, Miller KJ. Int. J. Mech. Sci. 20: 20106(1978)

25. Rice J. Mathematical Statistics and Data Analysis. Duxbury Press.( 1994)

26. ASTM E-8M-98. ( 1995)

27. ASTM E-466. (2004)

28. Lacerda JCD, Martins GD, Signoretti VT, Teixeira RLP. Int. J. Fatigue. 102:143-148(2017)

29. Shahzad M, Chaussumier M, Chieragatti R, Mabru C, et al. J. Mat. Processing. Tech. 210:1821-1826( 2010) 